\title{
Effect of liposomal fluidity on skin permeation of sodium fluorescein entrapped in liposomes
}

\author{
This article was published in the following Dove Press journal: \\ International Journal of Nanomedicine \\ 17 July 2015 \\ Number of times this article has been viewed
}

\author{
Thirapit Subongkot ${ }^{1}$ \\ Tanasait Ngawhirunpat ${ }^{2}$ \\ 'Department of Pharmaceutical \\ Technology, Faculty of Pharmaceutical \\ Sciences, Burapha University, \\ Chonburi, Thailand; ${ }^{2}$ Department of \\ Pharmaceutical Technology, Faculty \\ of Pharmacy, Silpakorn University, \\ Nakhon Pathom, Thailand
}

\begin{abstract}
The purpose of this study was to investigate the effect of ultradeformable liposome components, Tween 20 and terpenes, on vesicle fluidity. The fluidity was evaluated by electron spin resonance spectroscopy using 5-doxyl stearic acid and 16-doxyl stearic acid as spin labels for phospholipid bilayer fluidity at the $\mathrm{C} 5$ atom of the acyl chain near the polar head group (hydrophilic region) and the $\mathrm{C} 16$ atom of the acyl chain (lipophilic region), respectively. The electron spin resonance study revealed that Tween 20 increased the fluidity at the $\mathrm{C} 5$ atom of the acyl chain, whereas terpenes increased the fluidity at the $\mathrm{C} 16$ atom of the acyl chain of the phospholipid bilayer. The increase in liposomal fluidity resulted in the increased skin penetration of sodium fluorescein. Confocal laser scanning microscopy showed that ultradeformable liposomes with terpenes increase the skin penetration of sodium fluorescein by enhancing hair follicle penetration.
\end{abstract}

Keywords: ultradeformable liposomes, terpenes, fluidity, electron spin resonance spectroscopy, confocal laser scanning microscopy

\section{Introduction}

Transdermal drug delivery systems utilize skin as a transportation route and offer many advantages, including avoidance of first-pass hepatic metabolism, sustained and controlled drug release, and improved patient compliance. However, the stratum corneum, the outermost skin layer, exhibits a rate-limiting step in regulating drug absorption into the skin. Various strategies have been used to increase drug absorption across the skin, such as microneedles, ${ }^{1}$ iontophoresis, ${ }^{2}$ sonophoresis, ${ }^{3}$ electroporation, ${ }^{4}$ microdermabrasion, ${ }^{5}$ microemulsion, ${ }^{6}$ niosomes, ${ }^{7}$ and liposomes. ${ }^{8-10}$ Ultradeformable liposomes (ULs), also called transfersomes, are a type of elastic vesicle, introduced by Cevc and Blume. ${ }^{11}$ ULs generally consist of phospholipids and surfactant as a membrane softening agent. Due to their flexibility, ULs fit through narrow pores approximately one-tenth of their diameter. ULs also penetrate as intact vesicles through the skin into the blood circulation without permanent disintegration. ${ }^{12}$ ULs effectively increase the skin penetration of drugs both in vitro and in vivo. ${ }^{13-15}$

Terpenes, a class of penetration enhancers obtained from natural sources, have successfully been used as skin penetration enhancers for percutaneous absorption enhancement in various types of liposomes, specifically invasomes, ${ }^{16,17}$ and ULs. ${ }^{18,19}$ Electron spin resonance (ESR), also known as electron paramagnetic resonance, is a spectroscopy technique used to study molecular mobility by characterizing the unpaired electron of free radicals, also called spin probes, in an extreme applied magnetic field. This technique has been used to study membrane fluidity, ${ }^{20,21}$ the skin penetration enhancement mechanism of penetration enhancers and nanocarriers, ${ }^{22,23}$ and antioxidant properties. ${ }^{24}$
Correspondence: Tanasait Ngawhirunpat Department of Pharmaceutical Technology, Faculty of Pharmacy, Silpakorn University, 6 Rajamankha Nai Road, Muang, Nakhon Pathom 73000,

Thailand

Tel +66 34255800

Fax +66 34255801

Email ngawhirunpat_t@su.ac.th 
According to our previous study, ${ }^{18}$ ULs consisting of terpenes as a skin penetration enhancer and Tween 20 as a terpene solubilizer significantly enhanced the skin penetration of sodium fluorescein $(\mathrm{NaFl})$. Since ULs with terpenes penetrated via transfollicular pathway as major skin penetration pathway, ${ }^{19}$ we suggested that ULs with terpenes increased $\mathrm{NaFl}$ penetration by penetrating through hair follicles to bypass stratum corneum. To develop more effective ULs with terpene formulations and to identify the penetration enhancement mechanism, it is necessary to investigate the correlation between percutaneous penetration enhancement and liposomal fluidity, including the molecular structure of ULs with terpenes, which do not yet exist.

Therefore, we selected ESR to investigate the vesicle fluidity and molecular arrangement of the membrane softening components, terpenes and Tween 20, in UL structures using 5- and 16-doxyl stearic acid (5-DSA and 16-DSA) as spin labels. These spin probes have been widely used to study the membrane fluidity of liposomes ${ }^{20,25}$ and niosomes. ${ }^{26}$ 5-DSA has a nitroxide radical moiety at the fifth carbon atom of the acyl chain, whereas 16-DSA has a nitroxide radical moiety at the 16th carbon atom of the acyl chain (Figure 1). These selected spin labels, 5-DSA and 16-DSA, oriented their molecules parallel to the phospholipid molecules in a bilayer structure, providing mobility parameters for fluidity detection affected by the incorporated components at the $\mathrm{C} 5$ and $\mathrm{C} 16$ atoms of the phospholipid acyl chains.<smiles>C=C(C)[C]1CC=C(C)CC1</smiles><smiles>CC1(C)CC2CCC1(C)OC2</smiles><smiles>CC(C)=CCCC(C)=CCO</smiles>

\section{D-limonene Cineole Geraniol}

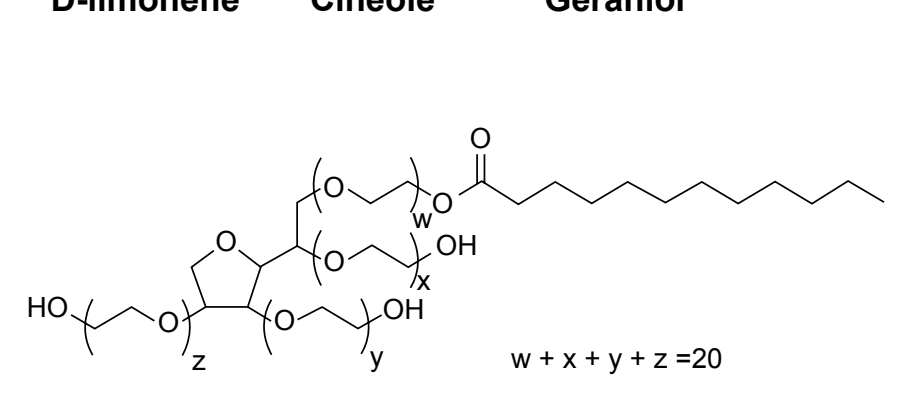

Tween 20
Because the follicular pathway is the major skin penetration pathway of ULs with terpenes, ${ }^{19}$ the mechanism of skin penetration enhancement by targeting the follicular penetration was elucidated. Liposomal vesicles were probed with rhodamine B 1,2-dihexadecanoyl-sn-glycero3-phosphoethanolamine triethylammonium salt (Rh-PE), which exhibits red fluorescence, whereas entrapped compound $(\mathrm{NaFl})$ exhibits green fluorescence. Confocal laser scanning microscopy (CLSM) using a co-localization technique was used to probe the skin penetration of fluorescentlabeled vesicles (UL-labeled Rh-PE) by comparing the fluorescence intensity and skin penetration depths between near follicular and nonfollicular regions. The objective of this study was to determine the correlation between liposomal fluidity and the increased in vitro skin penetration of $\mathrm{NaFl}$ and to elucidate the effect of vesicle fluidity on the follicular penetration enhancement of liposomes.

\section{Materials and methods}

\section{Materials}

Non-hydrogenated egg phosphatidylcholine (PC) (Coatsome NC-50; PC purity $\geq 95 \%$ ) was purchased from NOF Corporation (Tokyo, Japan). Cholesterol (Chol) was purchased from Sigma-Aldrich, St Louis, MO, USA. Tween 20 was purchased from Ajax Finechem (Auckland, New Zealand). Sodium fluorescein $(\mathrm{NaFl})$, d-limonene, 1,8-cineole, and geraniol were purchased from Sigma-Aldrich. 5-DSA and 16-DSA were purchased from Sigma-Aldrich. Lissamine ${ }^{\mathrm{TM}}$ rhodamine B 1,

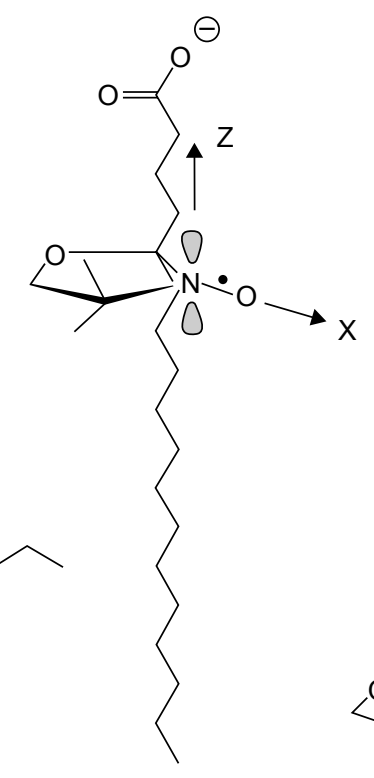

5-DSA

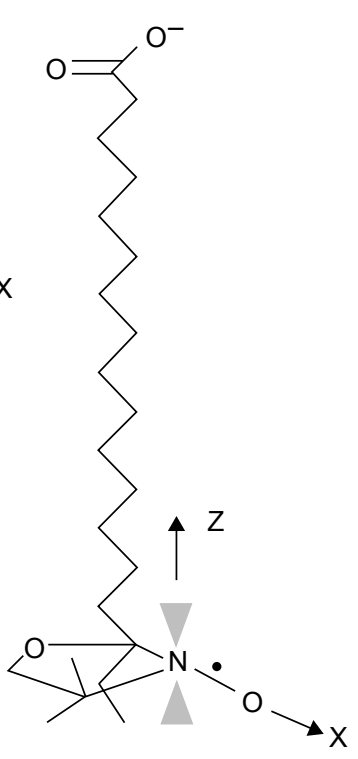

16-DSA

Figure I Chemical structures of the terpenes, Tween 20, 5-doxyl stearic acid (5-DSA) and I6-doxyl stearic acid (I6-DSA). 
2-dihexadecanoyl-sn-glycero-3-phosphoethanolamine triethylammonium salt (Rh-PE) was purchased from Invitrogen, CA, USA. All other reagents were of analytical grade and were commercially available.

\section{Preparation of UL containing monoterpenes}

The formulations of the liposomes containing PC, Chol, Tween 20, NaFl, and terpenes are shown in Table 1. The liposomes were prepared using the sonication method. A mixture of PC and Chol was dissolved in chloroform and methanol $(2: 1 \mathrm{v} / \mathrm{v})$. $\mathrm{NaFl}$ solution was prepared by dissolving $\mathrm{NaFl}$ to phosphate-buffered saline (PBS) at $\mathrm{pH} 7.4$ as a stock solution. The mixture of $\mathrm{PC}$ and Chol was then evaporated using a stream of nitrogen until a thin and homogeneous lipid film was formed. The thin film was placed in a desiccator connected to a vacuum pump for at least 6 hours. Then, the dried thin film was hydrated with PBS and NaFl solution. Tween 20 and each terpene were then added to the liposomal dispersion before sonication using a probe sonicator (Vibracell ${ }^{\mathrm{TM}}$, VCX 130 PB; Sonics \& Materials, Inc, Newtowns, CT, USA) for 30 minutes to reduce the size of the liposomes.

Conventional liposomes (CLs) and ULs were prepared using the same process as UL containing monoterpenes.

\section{Liposomal characterization}

Each liposomal formulation was diluted with distilled water prior to the measurement of size, zeta potential, and size distribution using a dynamic light scattering particle size analyzer (Zetasizer Nano-ZS; Malvern Instruments, Malvern, UK) with a $4 \mathrm{~mW}$ He-Ne laser at a scattering angle of $173^{\circ}$. All measurements were performed under ambient conditions and in triplicate.

\section{Assessment of liposomal fluidity using ESR}

All liposomal formulations were probed with stearic acid spin labels (5- or 16-DSA) using a spin label (5-DSA/16-DSA)-tolipid ratio of 1:100 M. Each spin label was dissolved in a mixture of chloroform and methanol $(2: 1 \mathrm{v} / \mathrm{v})$ before being added to a test tube of PC and Chol, followed by thin film formation as described in section Preparation of UL containing monoterpenes. All other ingredients were added except NaFl.

Liposomes probed with each spin label (5- or 16-DSA) were added to a glass capillary tube and sealed with Parafilm ${ }^{\circledR}$ before the ESR experiments. An ESR spectrum was recorded on a model JES-RE2X (JEOL, Tokyo, Japan) equipped with cylindrical cavity resonator and operated in $\left(\mathrm{TE}_{101}\right)$ mode. The ESR spectrometer was equipped with a microwave unit $\mathrm{X}$ band with a frequency of $8.8-9.6 \mathrm{GHz}$. The operating conditions of the equipment were microwave power of $1 \mathrm{~mW}$, modulation frequency of $100 \mathrm{kHz}$, modulation amplitude of $2.5 \times 100 \mathrm{mT}$, magnetic field scan of $1 \times 10 \mathrm{mT}$, sweep time of 30 seconds, detector time constant of $30 \mathrm{~ms}$, and temperature of $24^{\circ} \mathrm{C}$.

For 5-DSA (Figure 2A), the liposomal fluidity was estimated from the outermost separation between the spectral extrema, the maximum hyperfine splitting $\left(2 \mathrm{~T}_{\|}^{\prime}\right)$. The value of $2 \mathrm{~T}^{\prime}$ reflects the motional profiles near the phospholipid polar head group of the lipid bilayer. To determine the motional profiles at the phospholipid acyl chain near the lipophilic region of the phospholipid bilayer, the rotational correlation time $\left(\tau_{\mathrm{c}}\right)$ obtained from the 16-DSA spectrum (Figure 2B) was used as the liposomal fluidity parameter. The $\tau_{\mathrm{c}}$ was calculated from the equation as follows: ${ }^{27}$

$$
\tau_{\mathrm{c}}=\left(6.5 \times 10^{-10}\right) W_{0}\left[\left(\frac{h_{0}}{h_{-1}}\right)^{0.5}-1\right]
$$

where $W_{0}$ is the width of the midfield line of the spectrum in Gauss $(\mathrm{G}), h_{0}$ is the height of the midfield line of the spectrum and $h_{-1}$ is the height of the highfield line (Figure 2B). The $2 \mathrm{~T}_{\|}^{\prime}$ and $\tau_{\mathrm{c}}$ increased with a decrease in fluidity.

\section{In vitro skin penetration study}

Abdominal neonatal porcine skin (death from natural causes after birth) was obtained from a local slaughter house in Nakhon Pathom province and was used as barrier membrane for this

Table I Composition of NaFl-loaded liposomal formulations

\begin{tabular}{lllllll}
\hline Formulation (code) & PC (\%w/v) & NaFI (\%w/v) & Chol (\%w/v) & Tween 20 (\%w/v) & Terpenes (\%w/v) & PBS (\%w/v) (mL) \\
\hline CL & 0.77 & 0.21 & 0.07 & - & - & ad I00 \\
UL & 0.77 & 0.21 & 0.07 & 2 & - & ad I00 \\
ULL & 0.77 & 0.21 & 0.07 & 2 & I & ad I00 \\
ULC & 0.77 & 0.21 & 0.07 & 2 & $\mathrm{I}$ & ad I00 \\
ULG & 0.77 & 0.21 & 0.07 & 2 & $\mathrm{I}$ & ad I00 \\
\hline
\end{tabular}

Abbreviations: Ad, add to; Chol, cholesterol; CL, conventional liposomes; NaFl, sodium fluorescein; PBS, phosphate-buffered saline; PC, phosphatidylcholine; UL, ultradeformable liposomes; ULC, UL with cineole; ULG, UL with geraniol; ULL, UL with d-limonene. 

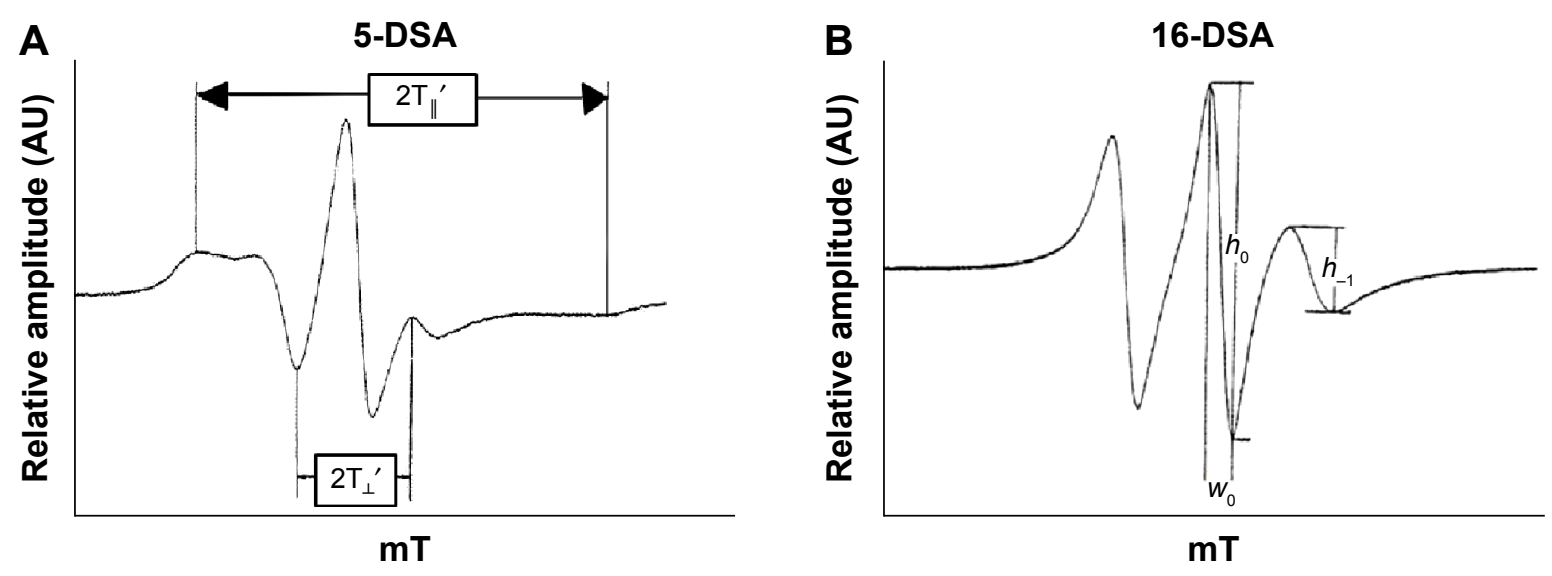

Figure 2 (A) ESR spectrum of 5-DSA and (B) ESR spectrum of 16-DSA.

Abbreviations: AU, arbitrary unit; 5-DSA, 5-doxyl stearic acid; 16-DSA, 16-doxyl stearic acid; ESR, electron spin resonance.

study. The subcutaneous fat was removed using medical scissors and surgical blades. The thickness of each skin membrane was approximately $0.6-0.7 \mathrm{~mm}$. The skin membranes were frozen at $-20^{\circ} \mathrm{C}$ until use. The membranes were thawed at room temperature using PBS prior to the experiments.

The experiment with $\mathrm{NaFl}$ penetration through porcine skin was performed using Franz diffusion cells with a penetration area of $2.31 \mathrm{~cm}^{2}$. Approximately $6.5 \mathrm{~mL}$ PBS was added to the receiver portion and stirred with magnetic bar at $500 \mathrm{rpm}$. The membrane was mounted between the donor and receiver portion with the stratum corneum facing the donor portion and the dermis facing the receiver medium. The diffusion cells were connected to a water circulating bath to maintain the temperature at $32^{\circ} \mathrm{C}$. Two milliliters of $\mathrm{NaFl}$ entrapped in the liposome formulation was added into the donor portion. At 1, 2, 4, 6, 8, and 24 hours, $0.5 \mathrm{~mL}$ of receiver medium was withdrawn for analysis, and an equal volume of PBS was added to the receiver portion to maintain a constant volume. Each sample was analyzed in triplicate.

The NaFl concentration was analyzed using a fluorescence spectroscopy method. One hundred microliters of the sample was pipetted into a black 96-well plate, and the fluorescence was detected in three replicates using a fluorescence spectrophotometer (Fusion ${ }^{\mathrm{TM}}$ Universal Microplate Analyzer; PACKARD Instrument Company, Inc, Downers Grove, IL, USA). The excitation wavelength was $485 \mathrm{~nm}$ and the emission wavelength was $535 \mathrm{~nm}$. The calibration curve for $\mathrm{NaFl}$ was in the range of $0.5-35 \mathrm{ng} / \mathrm{mL}$.

\section{CLSM study}

CLSM was used to evaluate the skin penetration differences between the follicular and nonfollicular regions of three liposomal formulations, CL, UL, and UL with $1 \%$ cineole, using a co-localization technique. The differences in the color of the fluorescent compounds were used to observe the vesicle penetration of the treated skin as follows: rhodamine (red)-probed liposomes, $\mathrm{NaFl}$ (green)-entrapped drug, and blue from skin autofluorescence.

The fluorescent probe (Rh-PE) was dissolved in a mixture of chloroform and methanol $(2: 1 \mathrm{v} / \mathrm{v})$ before addition to a test tube of $\mathrm{PC}$ and Chol mixture at a ratio of PC:Rh-PE of 100:1 M. The mixture of fluorescent probe and lipid (PC and Chol) was then evaporated using nitrogen gas to form thin film. Non-entrapped $\mathrm{NaFl}$ was separated from Rh-PE probed liposomes using a filtration technique with Amicon ${ }^{\circledR}$ Ultra-0.5 centrifugal devices (Millipore Corporation, Billerica, MA, USA). The Rh-PE probed liposomes were added to an ultrafiltration tube with a molecular weight cutoff of 3,000 Da and centrifuged at $4^{\circ} \mathrm{C}$ at $14,000 \times g$ for 30 minutes. The non-entrapped $\mathrm{NaFl}$ in the filtrate was removed. Then, the retentates device was turned upside down into a new concentrate collection tube and centrifuged at $4^{\circ} \mathrm{C}$ at $1,000 \times g$ for 2 minutes to transfer the entrapped NaFl-loaded Rh-PE probed liposomes from the device to the tube. The obtained sample was immediately used for the skin penetration study.

The skin penetration study of the NaFl-loaded Rh-PE probed liposomes was performed using Franz diffusion cells under the same conditions as described in the skin permeation study. The donor compartment was placed with $150 \mu \mathrm{L}$ of the entrapped NaFl-loaded Rh-PE probed liposomes. At 4 hours, each porcine skin was removed from diffusion cells, washed with PBS at least twice and stored at $-20^{\circ} \mathrm{C}$ prior to the CLSM investigation.

To evaluate the skin penetration of liposomes between the follicular and nonfollicular regions, each treated skin was placed on a $22 \times 50 \mathrm{~mm}$ cover slip (MENZEL-GLÄSER ${ }^{\circledR}$, 
Braunschweig, Germany) and then visualized with the $10 \times$ objective lens of an inverted Zeiss LSM 510 META microscope (Carl Zeiss Meditec AG, Jena, Germany), equipped with a He-Ne 1 laser (excitation wavelength $=543 \mathrm{~nm}$, emission wavelength $=580 \mathrm{~nm}$ ), Ar laser (excitation wavelength $=488$ $\mathrm{nm}$, emission wavelength $=514 \mathrm{~nm}$ ), and diode laser (excitation wavelength $=358 \mathrm{~nm}$, emission wavelength $=461 \mathrm{~nm}$ ) for Rh-PE, NaFl, and skin autofluorescence, respectively. These skin regions were scanned using a $20 \times$ objective lens to obtain $\mathrm{x}-\mathrm{z}$ plane images, by which the laser could scan through the tissue to compare the skin penetration depths and fluorescence intensity of the entrapped drug and liposomes at the follicular and nonfollicular regions.

\section{Data analysis}

The cumulative amount of $\mathrm{NaFl}$ penetrating the skin per unit area was plotted as a function of time. The skin penetration parameter (flux) was determined from the slope of the linear portion. All data were statistically analyzed using Student's $t$-test and analysis of variance. Differences of $P<0.05$ were considered statistically significant.

\section{Results and discussion Liposomal characterization}

The average size of the liposomes ranged from 39 to $98 \mathrm{~nm}$, with a narrow size distribution (polydispersity index $<0.4$ ) as shown in Table 2. The average size of the CLs was significantly larger than that of the ULs and ULs with terpenes. The average size of the ULs was significantly greater than that of the ULs with terpenes. There were no significant differences for the average size of the ULs with terpenes. These results indicate that the addition of Tween 20 and terpenes results in particle size reduction. Tasi et $\mathrm{al}^{28}$ reported that the surfactants exposed from the outer layer membrane increase the liposome particle curvature, while surfactants exposed to the inner leaflet do the opposite. Thus, we suggested that the

Table 2 Characterization parameters of different liposomal formulations

\begin{tabular}{llll}
\hline $\begin{array}{l}\text { Liposomal } \\
\text { formulations }\end{array}$ & $\begin{array}{l}\text { Particle size } \\
(\mathbf{n m})\end{array}$ & $\begin{array}{l}\text { Zeta potential } \\
(\mathbf{m V})\end{array}$ & $\begin{array}{l}\text { Polydispersity } \\
\text { index }\end{array}$ \\
\hline $\mathrm{CL}$ & $98.41 \pm 0.65$ & $-4.70 \pm 1.03$ & $0.266 \pm 0.002$ \\
UL & $52.43 \pm 0.23$ & $-13.21 \pm 0.9$ & $0.384 \pm 0.004$ \\
ULL & $43.82 \pm 0.48$ & $-10.30 \pm 0.2$ & $0.254 \pm 0.010$ \\
ULC & $43.70 \pm 0.61$ & $-8.23 \pm 0.31$ & $0.181 \pm 0.011$ \\
ULG & $42.79 \pm 0.94$ & $-10.75 \pm 3.02$ & $0.280 \pm 0.016$ \\
\hline
\end{tabular}

Note: Each value represents the mean \pm SD $(n=3)$.

Abbreviations: $\mathrm{CL}$, conventional liposomes; SD, standard deviation; UL, ultradeformable liposomes; ULC, UL with cineole; ULG, UL with geraniol; ULL, UL with d-limonene. decrease of liposomal size by terpenes may occur with the same mechanism as liposomal size reduction by surfactant addition. A reduction in liposomal size could possibly be attributed to a steric repulsion among terpenes molecules, which is exposed from the outer and inner bilayer membranes of liposomes. Addition of terpenes, therefore, reduced the liposomal size because there were more terpenes existing in the outer layer than in the inner bilayer membranes. The zeta potentials of all liposome formulations were negative $(-4.7$ to $-13.2 \mathrm{mV})$. PC is a zwitterionic compound with an isoelectric point between 6 and $6.7 .{ }^{29}$ Under the study conditions ( $\mathrm{pH} 7.4$ ), in which the $\mathrm{pH}$ was higher than the isoelectric point, the PC vesicles had an overall negative charge. Several papers reported that non-ionic surfactant-loaded liposomes ${ }^{30}$ and invasomes ${ }^{17}$ (terpenes-loaded liposomes) exhibit negative zeta potential ( -20 to $-30 \mathrm{mV}$ and -13 to $-14 \mathrm{mV}$, respectively) similar to our results. These negatively charged liposomal formulations also showed good physical and chemical stability, indicating that these particles had high zeta potential enough for electrostatic stabilization. Polydispersity index of all liposome formulations was less than 0.4 indicating a narrow size distribution of these liposomes.

\section{Liposomal fluidity}

Table 3 shows the ESR parameters $\left(2 \mathrm{~T}_{\|}^{\prime}\right.$ and $\left.\tau_{\mathrm{c}}\right)$ of the different liposomal formulations. The $2 \mathrm{~T}_{\|}^{\prime}$ obtained from the 5-DSA was used to detect the motional profiles near the polar head group of the phospholipid acyl chain, whereas the $\tau_{\mathrm{c}}$ obtained from the 16-DSA was used to detect the motional profiles at the end of the lipophilic chain. The $2 \mathrm{~T}_{\|}^{\prime}$ of the ULs was significantly lower than that of the CLs, whereas the $\tau_{\mathrm{c}}$ values were not significantly different between the CLs and the ULs. It is concluded that the addition of Tween 20 to the ULs did not increase the fluidity of the acyl chain near the hydrophobic region of the phospholipid bilayer; however, it increased the fluidity of the acyl chain near

Table 3 ESR parameters (maximum hyperfine splitting and rotational correlation time) and in vitro skin penetration parameter (flux) of each liposomal formulation

\begin{tabular}{|c|c|c|c|}
\hline $\begin{array}{l}\text { Liposomal } \\
\text { formulations }\end{array}$ & $2 T_{\|}^{\prime}(m T)$ & $\tau_{\mathrm{c}}(\mathrm{ns})$ & $\begin{array}{l}\text { Flux } \\
\left(\mu g / \mathrm{cm}^{2} / \mathrm{h}\right)\end{array}$ \\
\hline$C L$ & $8.19 \pm 0.24$ & $1.76 \pm 0.07$ & $0.0137 \pm 0.0081$ \\
\hline UL & $4.70 \pm 0.01$ & $1.32 \pm 0.01$ & $0.0611 \pm 0.0163$ \\
\hline ULL & $4.7 I \pm 0.25$ & $0.704 \pm 0.001$ & $0.4876 \pm 0.0962$ \\
\hline ULC & $4.68 \pm 0.04$ & $0.7|9 \pm 0.07|$ & $0.4653 \pm 0.1472$ \\
\hline ULG & $4.89 \pm 0.06$ & $0.629 \pm 0.022$ & $0.4073 \pm 0 .|42|$ \\
\hline
\end{tabular}

Note: Each value represents the mean $\pm S D(n=3)$.

Abbreviations: CL, conventional liposomes; ESR, electron spin resonance; SD, standard deviation; UL, ultradeformable liposomes; ULC, UL with cineole; ULG, UL with geraniol; ULL, UL with d-limonene. 
the polar head group of the phospholipid bilayer. This result also indicates that Tween 20 molecules are localized near the polar head group of the liposomal bilayer. The $\tau_{\mathrm{c}}$ of each ULs with different terpenes, ULL, ULC, and ULG, was significantly decreased compared to that of the ULs, whereas the $2 \mathrm{~T}_{\|}^{\prime}$ values of the ULs and ULs with terpenes were not significantly different. Therefore, terpenes increased the fluidity at the C16 atom of the phospholipid acyl chain of the vesicle bilayer. This result also indicates that terpene molecules are localized in the phospholipid acyl chain near the lipophilic region of the vesicle bilayer. Of the ULs with different terpenes (ULL, $\mathrm{ULC}$, and ULG), the $2 \mathrm{~T}_{\|}^{\prime}$ and $\tau_{\mathrm{c}}$ values were not significantly different. We conclude that different types of monoterpenes incorporated in the ULs did not affect vesicle fluidity. Our findings are consistent with those of Dragicevic-Curic et $\mathrm{al}^{25}$ who showed that the addition of $1 \%$ terpene/terpene mixture to invasomes significantly increased the vesicle fluidity around the $\mathrm{C} 16$ atom of the phospholipid acyl chain.

\section{In vitro skin penetration study}

The flux of different liposomal formulations is shown in Table 3. The flux of NaFl from ULs was significantly higher than that of CLs. The flux of NaFl from ULs with different terpenes (ULL, ULC, and ULG) was significantly higher than that from ULs. The flux among ULL, ULC, and ULG was not significantly different.

Because the $2 \mathrm{~T}_{\|}^{\prime}$ values between ULs and ULs with terpenes were not significantly different, $\tau_{\mathrm{c}}$ was selected as a candidate of liposomal fluidity to assess the correlation between fluidity and skin penetration enhancement. From the results of flux and $\tau_{\mathrm{c}}$ (Table 3 ), the flux of $\mathrm{NaFl}$ from ULs was significantly higher than that of CLs, whereas the $\tau_{\mathrm{c}}$ of ULs was significantly lower than that of CLs. The flux of $\mathrm{NaFl}$ from ULs with different terpenes was significantly higher than that of ULs, whereas the $\tau_{\mathrm{c}}$ of ULs with different terpenes was significantly lower than that of ULs. There were no significant differences for the flux of ULs with different terpenes, and their $\tau_{\mathrm{c}}$ values were also not significantly different. These results indicate that the decrease in $\tau_{\mathrm{c}}$ correlated with the increase in $\mathrm{NaFl}$ flux. We conclude that the increase in liposomal fluidity results in increased skin penetration of $\mathrm{NaFl}$. According to the invasome fluidity assessment using ESR by Dragicevic-Curic et $\mathrm{al}^{25}$ there was no direct correlation between invasome fluidity and skin penetration ability.

\section{Molecular structure elucidation}

The fluidity assessment using ESR elucidated the molecular structure of ULs with terpenes. From our results, the addition of Tween 20 increased the vesicle fluidity near the polar head group of the phospholipid bilayer. Tween 20 or polyoxyethylene (20) sorbitan monolaurate is a non-ionic surfactant consisting of a polyoxyethylene group as the hydrophilic portion and a hydrocarbon chain of lauric acid as the lipophilic portion. We hypothesize that Tween 20 molecules intercalated between the phospholipid molecules by turning their hydrophilic portion toward the phosphate group, as shown in Figure 3. For terpenes, there was an increase in vesicle fluidity near the $\mathrm{C} 16$ atom of the phospholipid acyl chain. Monoterpenes (d-limonene, cineole, and geraniol) added to ULs with different terpenes are small lipophilic molecules. Therefore, terpenes were localized near the end of the phospholipid acyl chain, as shown in Figure 3.

\section{CLSM study}

Our previous study ${ }^{19}$ found that UL with terpenes increased skin penetration by penetrating through the hair follicle as the primary penetration pathway. Therefore, we tested our hypothesis that ULs with terpenes increase the skin penetration of $\mathrm{NaFl}$ by enhancing the follicular penetration to bypass the stratum corneum. Three liposomal formulations were selected to study the follicular penetration enhancement. CLSM was used to visualize the skin penetration of the entrapped drug and liposomes between the near follicular and nonfollicular regions of CLs, ULs, and ULs with $1 \%$ cineole (as candidate ULs with terpenes) using a multifluorescence compound technique. Both skin penetration depths and fluorescence intensity were compared to evaluate the follicular penetration enhancement.

\section{CLSM of skin treated with CLs}

Top view images of sequential follicular and nonfollicular regions from the same skin tissue at 4 hours are shown in Figure 4A1 and B1, respectively. The marked areas of these images (Figure 4A1 and B1) were scanned to obtain the greatest penetration depths and fluorescence intensity of $\mathrm{NaFl}$ and CLs. The $\mathrm{x}-\mathrm{z}$ plane serial optical images from different skin depths at the follicular and nonfollicular regions are shown in Figure 4A2 and B2. The merge of $\mathrm{x}-\mathrm{z}$ plane serial optical images from different skin depths at the follicular and nonfollicular regions are shown in Figure 4A3 and B3, respectively. Both $\mathrm{NaFl}$ and CLs penetrate through the follicular region and the nonfollicular region with the same distance of only approximately $55 \mu \mathrm{m}$.

Figure 4C shows the fluorescence intensity of $\mathrm{NaFl}$ (green fluorescence) and CLs (red fluorescence) at different penetration depths from follicular and nonfollicular regions. The fluorescence intensity of $\mathrm{NaFl}$ at the follicular region was not different from the nonfollicular region. The fluorescence 


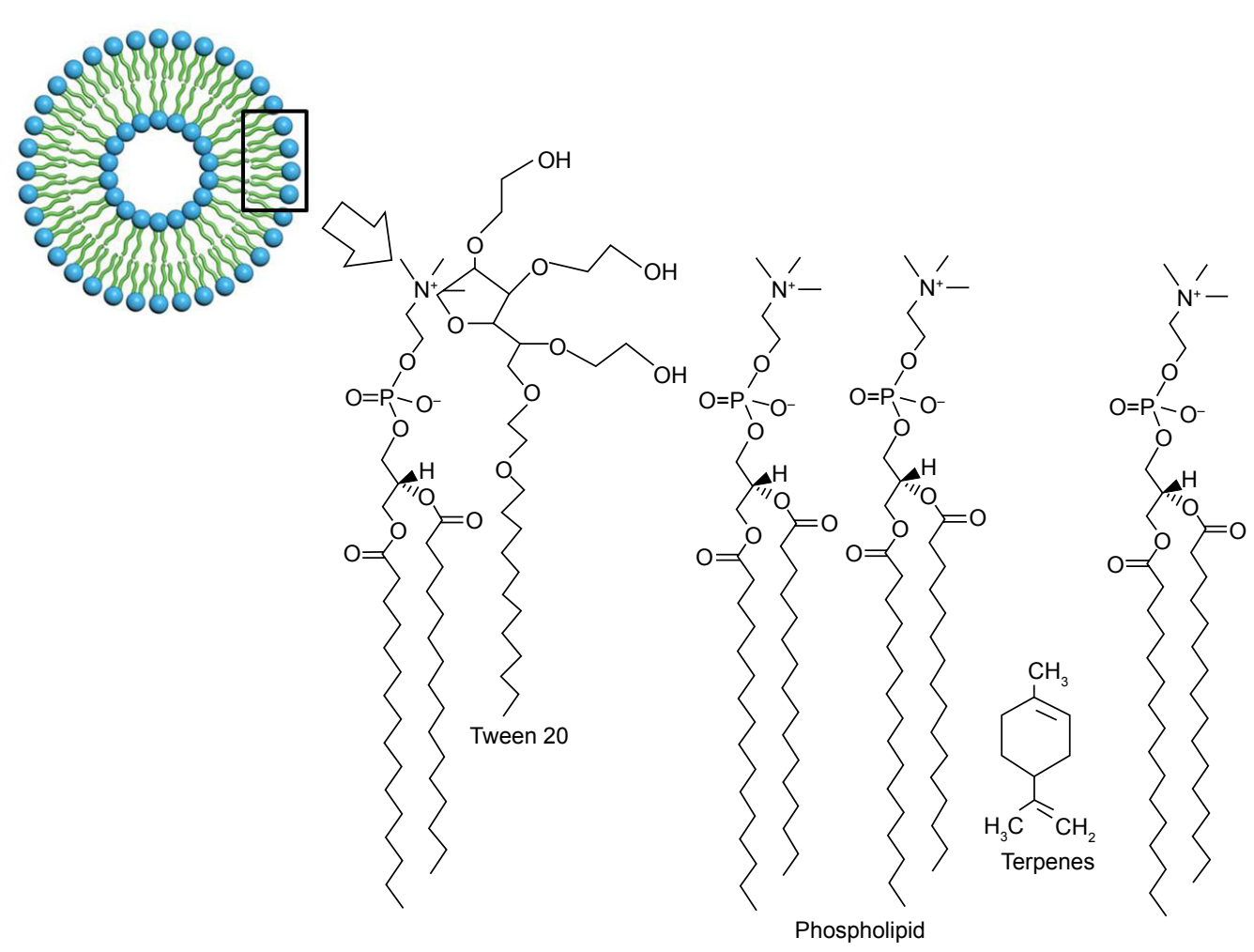

Figure 3 Molecular structure of ultradeformable liposomes with terpenes.

intensity of Rh-PE was also not different between the follicular and nonfollicular regions. These results indicate that CLs penetrated through the follicular region to a similar extent as in the nonfollicular region.

\section{CLSM of skin treated with ULs}

Top view images of follicular and nonfollicular regions from the same skin tissue at 4 hours are shown in Figure 5A1 and B1, respectively. The marked areas of these images (Figure 5A1 and B1) were scanned to obtain the greatest penetration depths and fluorescence intensity of NaFl and ULs. The $\mathrm{x}-\mathrm{z}$ plane serial optical images from different skin depths at the follicular and nonfollicular regions are shown in Figure $5 \mathrm{~A} 2$ and B2, respectively. The merge of $\mathrm{x}-\mathrm{z}$ plane serial optical images from different skin depths at the follicular and nonfollicular regions are shown in Figure 5A3 and B3, respectively Both $\mathrm{NaFl}$ and ULs penetrated through the follicular region and the nonfollicular region with the same distance of approximately $75 \mu \mathrm{m}$.

Figure 5C shows the fluorescence intensity of $\mathrm{NaFl}$ (green fluorescence) and ULs (red fluorescence) at different penetration depths from follicular and nonfollicular regions. The fluorescence intensity of $\mathrm{NaFl}$ at the nonfollicular region was greater than the follicular region in the beginning distance $(0-30 \mu \mathrm{m})$. However, for the other distance $(30-115 \mu \mathrm{m})$, the fluorescence intensity of $\mathrm{NaFl}$ was not different between the follicular and nonfollicular regions. The fluorescence intensity of Rh-PE was not different between the follicular and nonfollicular regions. Our results indicate that ULs penetrated through the follicular region to a similar extent as the nonfollicular region.

\section{CLSM of skin treated with ULs with I\% cineole}

Top view images of follicular and nonfollicular regions from the same skin tissue are shown in Figure 6A1 and B1, respectively. The marked areas of these images (Figure 6A1 and B1) were scanned to obtain the greatest penetration depths and fluorescence intensity of NaFl-loaded ULs with $1 \%$ cineole labeled $\mathrm{Rh}-\mathrm{PE}$. The gallery of $\mathrm{x}-\mathrm{z}$ plane serial optical images from different skin depths at follicular and nonfollicular regions is shown in Figure 6A2 and B2, respectively. The merge of $\mathrm{x}-\mathrm{z}$ plane serial optical images from different skin depths at the follicular and nonfollicular regions are shown in Figure 6A3 and B3, respectively. $\mathrm{NaFl}$ and liposomes penetrated to $90 \mu \mathrm{m}$ in both follicular and nonfollicular regions.

Figure $6 \mathrm{C}$ shows the fluorescence intensity of $\mathrm{NaFl}$ (green fluorescence) and liposomes (red fluorescence) at different penetration depths from the follicular and nonfollicular regions. The fluorescence intensity of $\mathrm{NaFl}$ between the follicular and nonfollicular regions was not different. However, the fluorescence intensity of Rh-PE at the follicular region 
A1

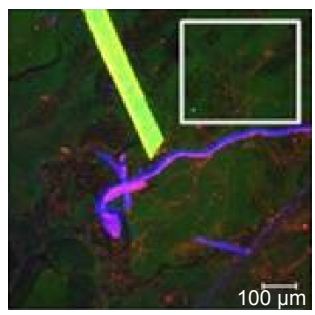

A2 (0-55 $\mu \mathrm{m})$

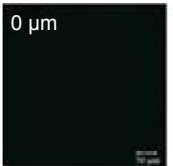

$20 \mu \mathrm{m}$

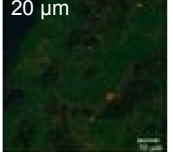

$40 \mu \mathrm{m}$

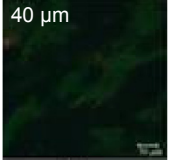

A3
B1

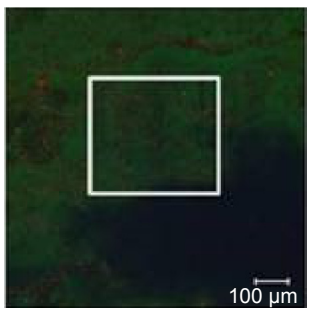

B2 (0-55 $\mu \mathrm{m})$
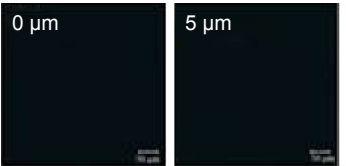

$10 \mu \mathrm{m}$

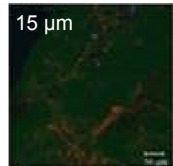

$35 \mu \mathrm{m}$

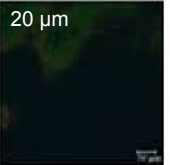

$25 \mu \mathrm{m}$
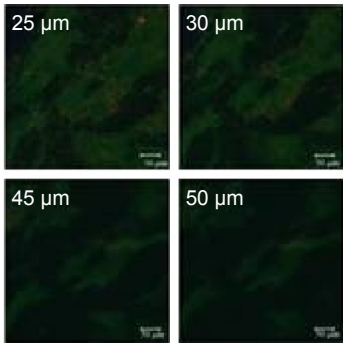

$50 \mu \mathrm{m}$

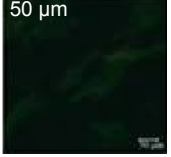

$55 \mu \mathrm{m}$

\begin{tabular}{l|l}
$40 \mu \mathrm{m}$ & $45 \mathrm{~m}$
\end{tabular}

$45 \mu \mathrm{m}$

$50 \mu \mathrm{m}$

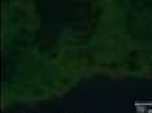

B3

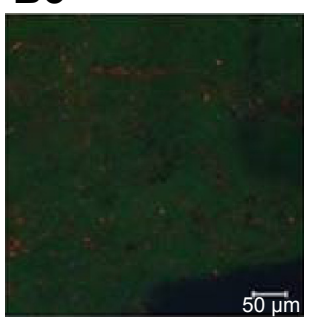

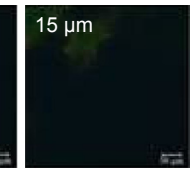

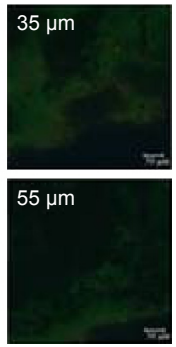

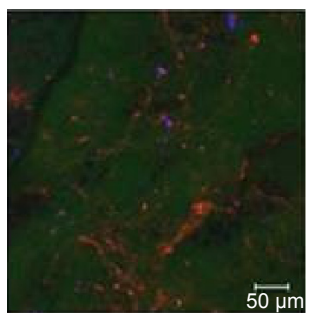

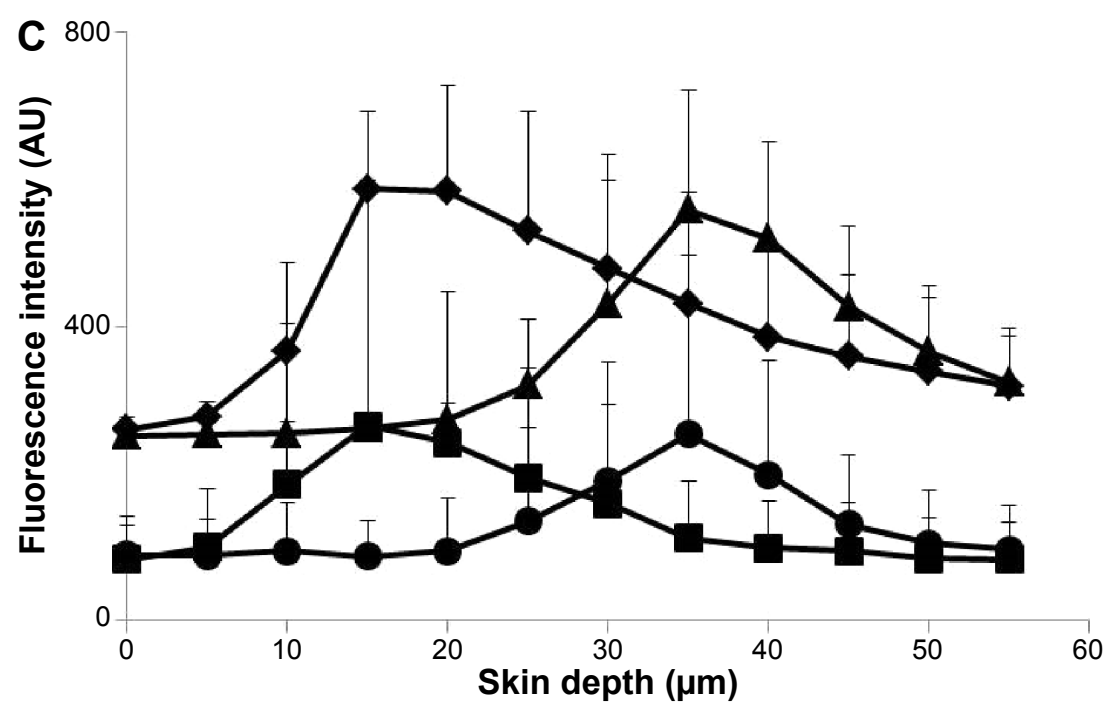

Figure 4 CLSM images of skin treated with CLs.

Notes: (AI and BI) The $\mathrm{x}-\mathrm{y}$ plane serial follicular and nonfollicular localization of porcine skin treated with NaFl-loaded Rh-PE-labeled CLs at 4 hours. The scale bar represents $100 \mu \mathrm{m}$. (A2 and B2) The serial $x-z$ plane magnification of the marked area from the follicular and nonfollicular regions at different skin depths using a $20 x$ objective lens. The scale bar represents $50 \mu \mathrm{m}$. (A3 and B3) The intensity over projection of z-axis images of A2 and B2, respectively. The scale bar represents $50 \mu \mathrm{m}$. The blue, green, and red fluorescence are the autofluorescence, $\mathrm{NaFl}$, and Rh-PE, respectively. (C) Comparison of fluorescence intensity profiles of $\mathrm{NaFl}$ and Rh-PE at different skin depths of $\mathbf{A} 2(\bullet, \mathrm{NaFl} ; \mathbf{\mathbf { a }}, \mathrm{Rh}-\mathrm{PE})$ and $\mathbf{B 2}(\mathbf{\Delta}, \mathrm{NaFl} ; \bullet, \mathrm{Rh}-\mathrm{PE})$.

Abbreviations: AU, arbitrary unit; CLs, conventional liposomes; CLSM, confocal laser scanning microscopy; NaFl, sodium fluorescein; Rh-PE, rhodamine B I,2-dihexadecanoylsn-glycero-3-phosphoethanolamine triethylammonium salt. 
A1

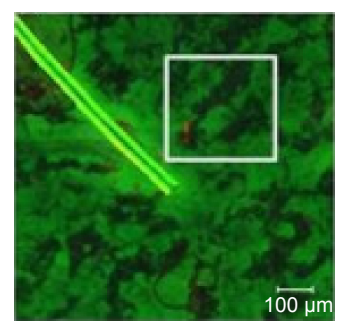

A2 $(0-75 \mu \mathrm{m})$
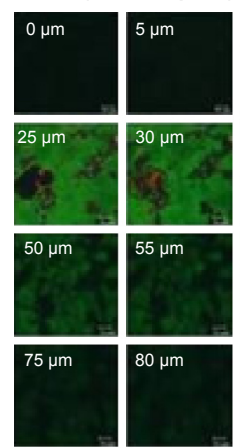

$100 \mu \mathrm{m}$

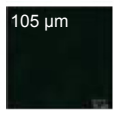

A3

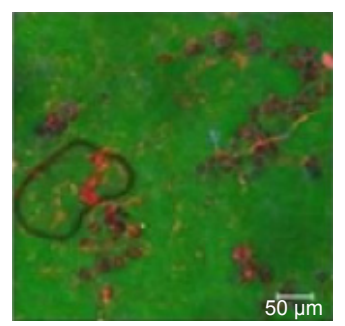

B1

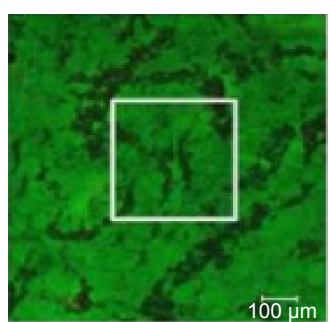

B2 $(0-75 \mu \mathrm{m})$
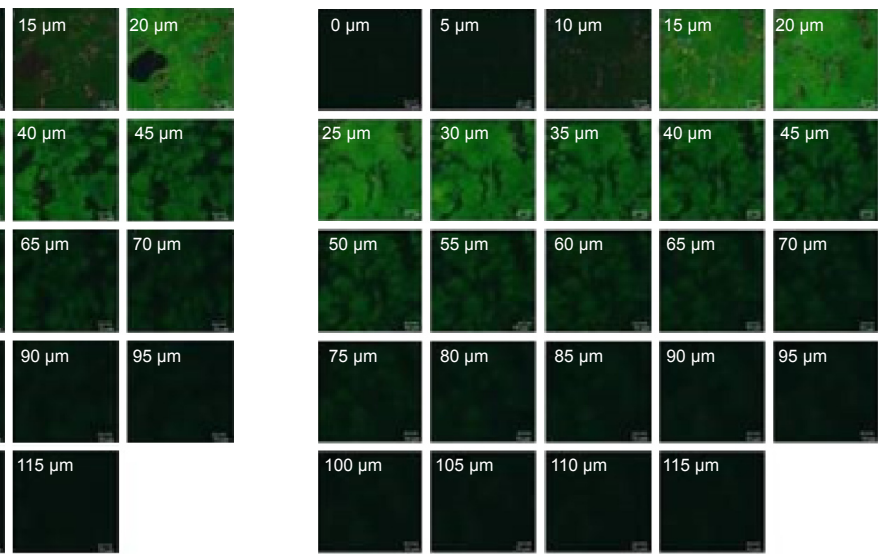

$75 \mu \mathrm{m}$
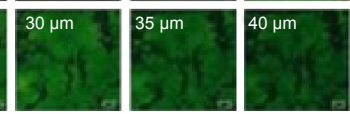

$45 \mu \mathrm{m}$
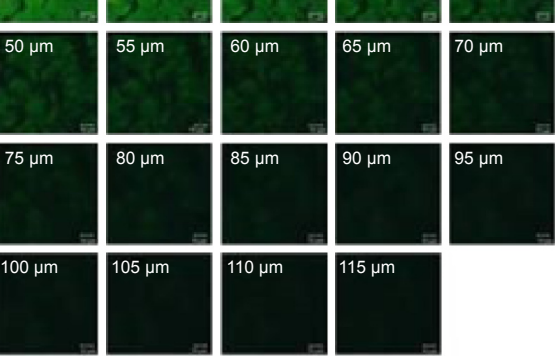

B3
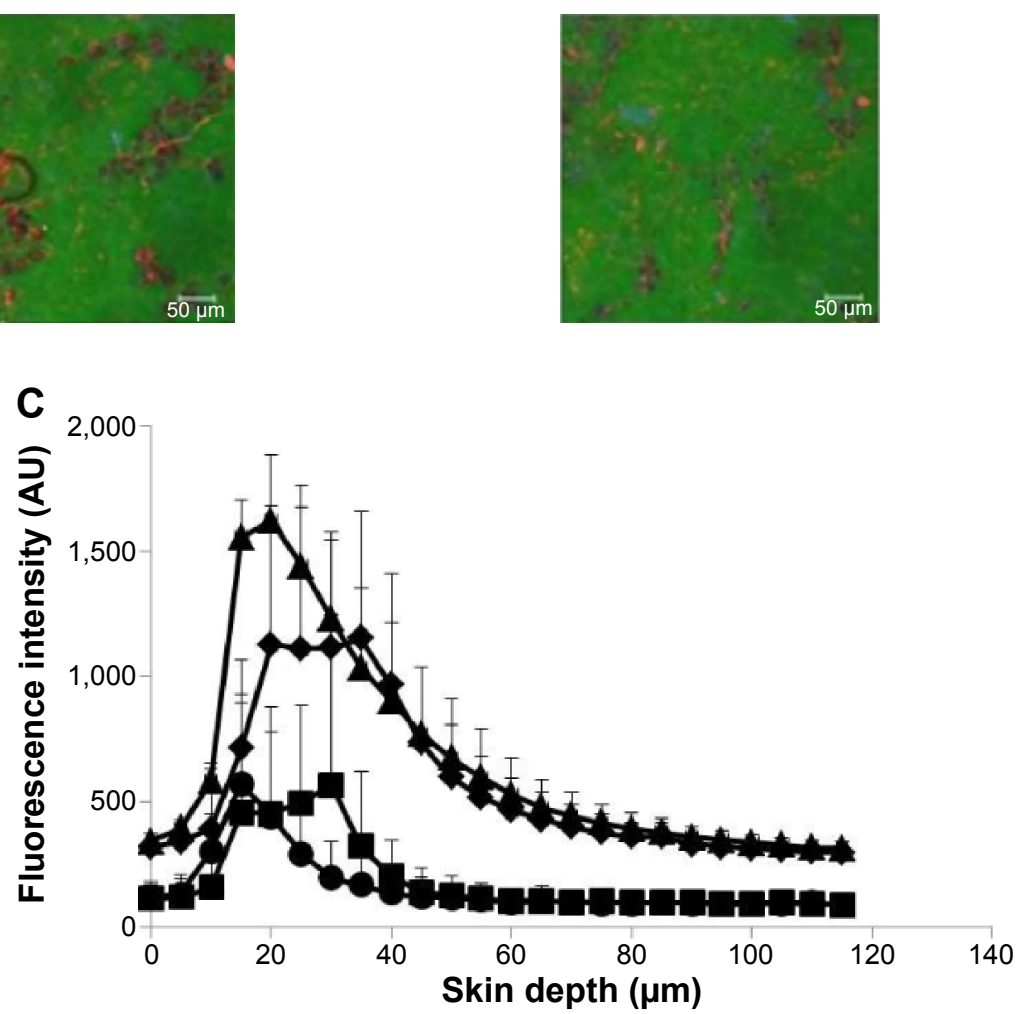

Figure 5 CLSM images of skin treated with ULs.

Notes: (AI and BI) The $x-y$ plane serial follicular and nonfollicular localization of porcine skin treated with NaFl-loaded Rh-PE-labeled UL at 4 hours. The scale bar represents $100 \mu \mathrm{m}$. (A2 and B2) The serial $\mathrm{x}-\mathrm{z}$ plane magnification of the marked area from the follicular and nonfollicular regions at different skin depths using a $20 \times$ objective lens. The scale bar represents $50 \mu \mathrm{m}$. (A3 and B3) The intensity over projection of the z-axis images of A2 and B2, respectively. The scale bar represents $50 \mu \mathrm{m}$. The blue, green, and red fluorescence are the autofluorescence, $\mathrm{NaFl}$, and Rh-PE, respectively. (C) Comparison of the fluorescence intensity profiles of $\mathrm{NaFI}$ and $\mathrm{Rh}-\mathrm{PE}$ at different skin depths of $\mathbf{A} 2(\bullet, \mathrm{NaFl} ; \mathbf{\bullet}, \mathrm{Rh}-\mathrm{PE})$ and $\mathbf{B} 2(\boldsymbol{\Delta}, \mathrm{NaFl} ; \bullet, \mathrm{Rh}-\mathrm{PE})$.

Abbreviations: AU, arbitrary unit; CLSM, confocal laser scanning microscopy; NaFl, sodium fluorescein; Rh-PE, rhodamine B I,2-dihexadecanoyl-sn-glycero-3phosphoethanolamine triethylammonium salt; ULs, ultradeformable liposomes. 
A1

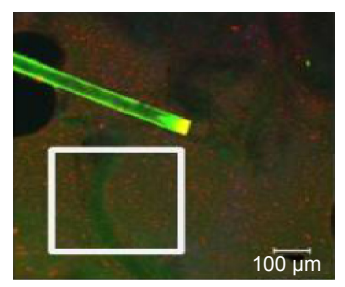

A2 $(0-90 \mu \mathrm{m})$
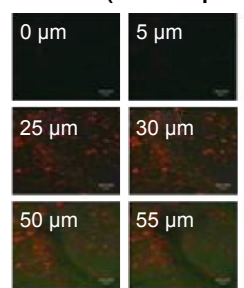

$75 \mu \mathrm{m}$

$100 \mu \mathrm{m}$

$100 \mu \mathrm{m}$

$125 \mu \mathrm{m}$
55 um

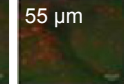

$80 \mu \mathrm{m}$

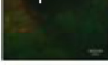

$105 \mu \mathrm{m}$

-

$130 \mu \mathrm{m}$

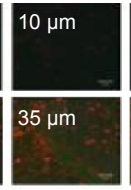

$60 \mu \mathrm{m}$

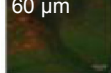

$85 \mu \mathrm{m}$

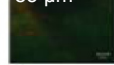

$110 \mu \mathrm{m}$

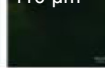

$135 \mu \mathrm{m}$

$115 \mu \mathrm{m}$

$140 \mu \mathrm{m}$

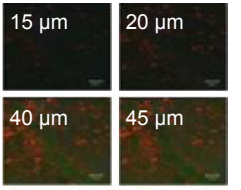

B2 $(0-90 \mu \mathrm{m})$
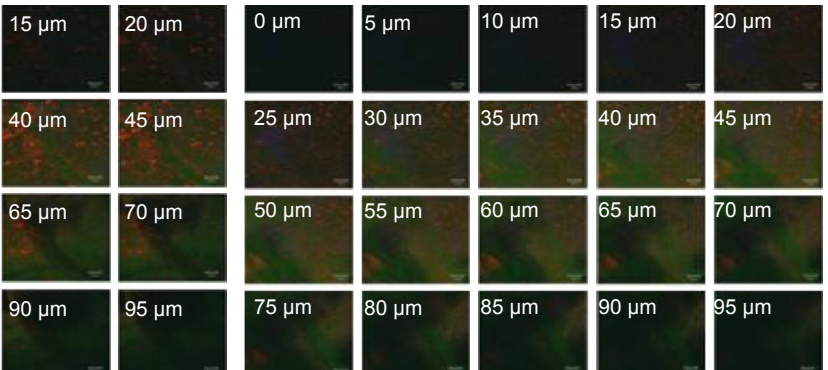

$95 \mu \mathrm{m}$
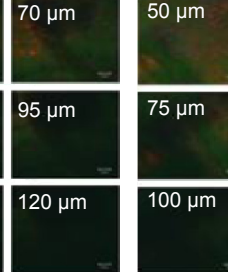

$75 \mu \mathrm{m}$
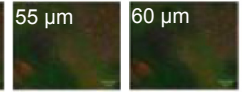

$65 \mu \mathrm{m}$
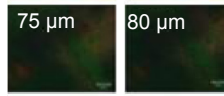

$85 \mu \mathrm{m}$

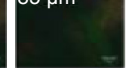

$120 \mu \mathrm{m}$
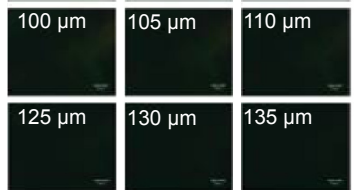

$125 \mu \mathrm{m}$

$130 \mu \mathrm{m}$
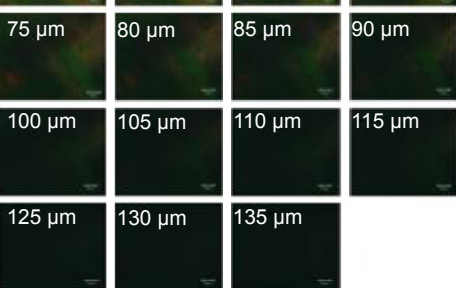

$115 \mu \mathrm{m}$
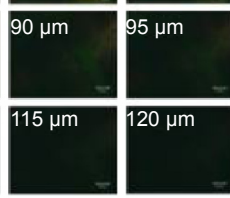

A3

B3
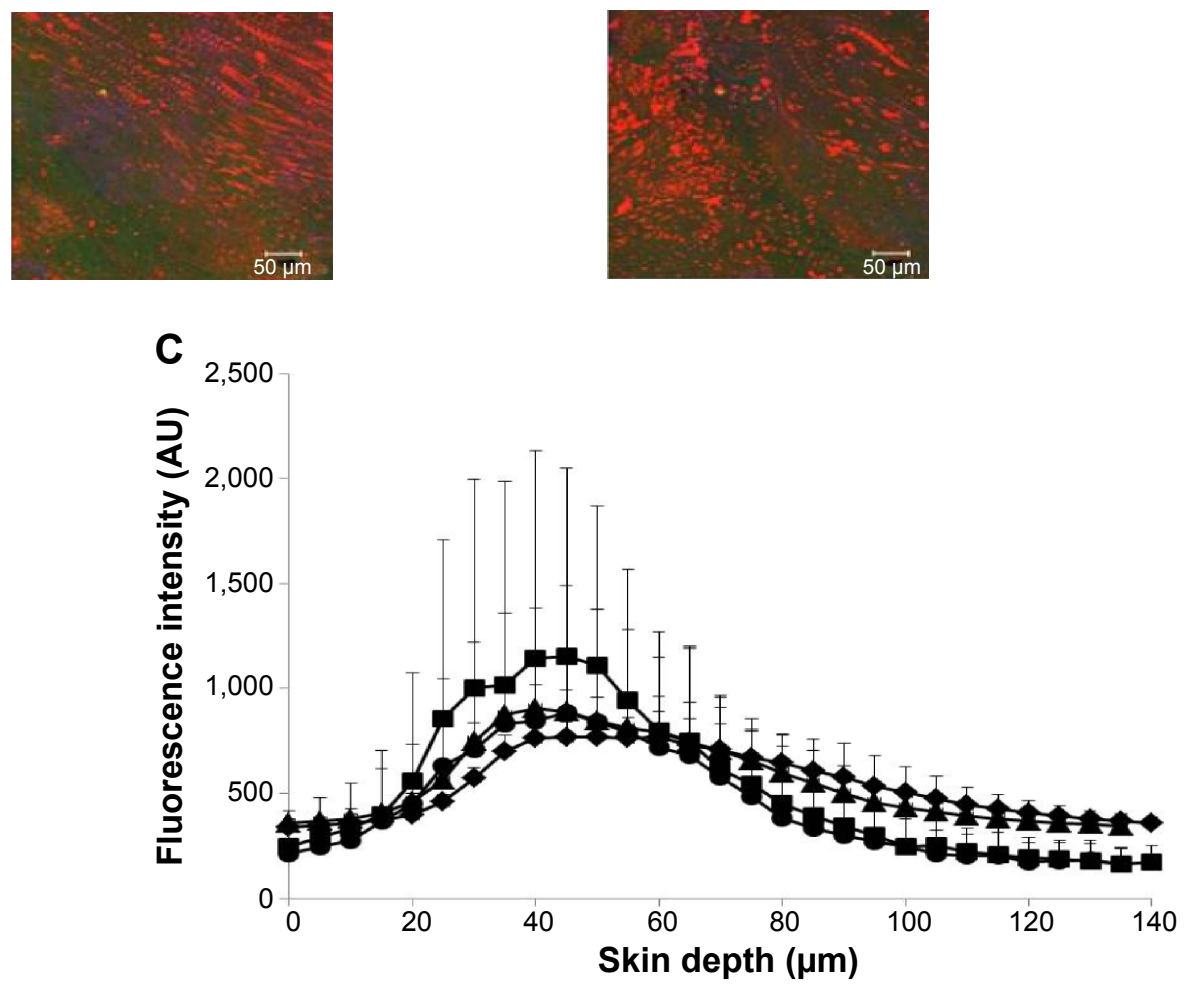

Figure 6 CLSM images of skin treated with ULs with $1 \%$ cineole.

Notes: (AI and BI) The $\mathrm{x}-\mathrm{y}$ plane serial follicular and nonfollicular localization of porcine skin treated with NaFl-loaded Rh-PE-labeled ULs with I\% cineole at 4 hours. The scale bar represents $100 \mu \mathrm{m}$. (A2 and B2) The serial x-z plane magnification of the marked area from the follicular and nonfollicular regions at different skin depths using a $20 \times$ objective lens. The scale bar represents $50 \mu \mathrm{m}$. (A3 and B3) The intensity over projection of z-axis images of A2 and B2, respectively. The scale bar represents $50 \mu \mathrm{m}$. The blue, green, and red fluorescence are the autofluorescence, $\mathrm{NaFl}$, and $\mathrm{Rh}-\mathrm{PE}$, respectively. (C) Comparison of the fluorescence intensity profiles of $\mathrm{NaFl}$ and Rh-PE at different skin depths of $\mathbf{A} 2(\bullet, \mathrm{NaFl} ; \mathbf{-}, \mathrm{Rh}-\mathrm{PE})$ and $\mathbf{B 2}(\boldsymbol{\Delta}, \mathrm{NaFl} ; \bullet \bullet, \mathrm{Rh}-\mathrm{PE})$.

Abbreviations: AU, arbitrary unit; CLSM, confocal laser scanning microscopy; NaFl, sodium fluorescein; Rh-PE, rhodamine B I,2-dihexadecanoyl-sn-glycero-3phosphoethanolamine triethylammonium salt; ULs, ultradeformable liposomes. 
was significantly higher than the nonfollicular region, particularly at penetration depths of $20-60 \mu \mathrm{m}$, indicating that ULs with $1 \%$ cineole penetrated through the follicular region more than the nonfollicular region. Three skin penetration pathways were proposed, intercellular, transcellular, and the transappendageal or transfollicular pathway. ${ }^{31}$ To overcome the stratum corneum barrier, follicular penetration is considered an effective penetration pathway because this pathway can bypass the stratum corneum to the dermis. ULs with $1 \%$ cineole increased the skin penetration of $\mathrm{NaFl}$ by penetrating via the follicular pathway.

The fluorescence intensity of $\mathrm{NaFl}$ in Figure 6A2 and B2 was lower than in Figure 5A2 and B2. This difference may be a result of the different vesicle release rates in the skin tissue. These CLSM studies reveal that the increase in skin penetration of $\mathrm{NaFl}$ from ULs with terpenes was caused by the selective follicular penetration of vesicles.

\section{Conclusion}

These studies revealed that the liposomal fluidity correlated with the skin penetration enhancement of the entrapped drug. The increase in liposomal fluidity resulted in the skin penetration enhancement of NaFl. Based on the ESR study, the addition of Tween 20 resulted in vesicle fluidity at the $\mathrm{C} 5$ atom of the phospholipid acyl chain, indicating that Tween 20 molecules were localized near the hydrophilic portion of the phospholipid bilayer. Terpenes, which were incorporated in ULs, induced liposomal fluidity at the C16 atom of the phospholipid acyl chain, indicating that terpene molecules were localized near the lipophilic region of the phospholipid bilayer. The CLSM study shows that the mechanism of skin penetration enhancement of $\mathrm{NaFl}$ from ULs with terpenes occurred because of an increase in the follicular penetration of phospholipid vesicles.

\section{Acknowledgments}

The authors gratefully acknowledge the Thailand Research Funds through the Basic Research Grant (Grant No 5680016), and the Faculty of Pharmacy, Silpakorn University, Nakhon Pathom, Thailand for facilities and financial support.

\section{Disclosure}

The authors report no conflicts of interest in this work.

\section{References}

1. Lee JW, Park JH, Prausnitz MR. Dissolving microneedles for transdermal drug delivery. Biomaterials. 2008;29:2113-2124.

2. Djabri A, Guy RH, Delgado-Charro MB. Transdermal iontophoresis of ranitidine: an opportunity in paediatric drug therapy. Int J Pharm. 2012; 435:27-32.
3. Herwadkar A, Sachdeva V, Taylor LF, Silver H, Banga AK. Low frequency sonophoresis mediated transdermal and intradermal delivery of ketoprofen. Int J Pharm. 2012;423:289-296.

4. Sung KC, Fang JY, Wang JJ, Hu OY. Transdermal delivery of nalbuphine and its prodrugs by electroporation. Eur J Pharm Sci. 2003;18:63-70.

5. Andrews SN, Zarnitsin V, Bondy B, Prausnitz MR. Optimization of microdermabrasion for controlled removal of stratum corneum. Int $J$ Pharm. 2011;407:95-104.

6. Hathout RM, Nasr M. Transdermal delivery of betahistine hydrochloride using microemulsions: physical characterization, biophysical assessment, confocal imaging and permeation studies. Colloids Surf B Biointerfaces. 2013;110:254-260.

7. El-Laithy HM, Shoukry O, Mahran LG. Novel sugar esters proniosomes for transdermal delivery of vinpocetine: preclinical and clinical studies. Eur J Pharm Biopharm. 2011;77:43-55.

8. Shen LN, Zhang YT, Wang Q, Xu L, Feng NP. Enhanced in vitro and in vivo skin deposition of apigenin delivered using ethosomes. Int $J$ Pharm. 2014:460:280-288.

9. El-Nabarawi MA, Bendas ER, El Rehem RT, Abary MY. Transdermal drug delivery of paroxetine through lipid-vesicular formulation to augment its bioavailability. Int J Pharm. 2013;443:307-317.

10. Cevc G, Blume G. Biological activity and characteristics of triamcinolone-acetonide formulated with the self-regulating drug carriers, Transfersome ${ }^{\circledR}$. Biochim Biophys Acta. 2003;1614:156-164.

11. Cevc G, Blume G. Lipid vesicles penetrate into intact skin owing to the transdermal osmotic gradients and hydration force. Biochim Biophys Acta. 1992;1104:226-232.

12. Cevc G, Schätzlein A, Richardsen H. Ultradeformable lipid vesicles can penetrate the skin and other semi-permeable barriers unfragmented: evidence from double label CLSM experiments and direct size measurements. Biochim Biophys Acta. 2002;1564:21-30.

13. Rother M, Seidel EJ, Clarkson PM, Mazgareanu S, Vierl U, Rother I. Efficacy of epicutaneous Diractin ${ }^{\circledR}$ (ketoprofen in Transfersome ${ }^{\circledR}$ gel) for the treatment of pain related to eccentric muscle contractions. Drug Des Devel Ther. 2009;3:143-149.

14. Cevc G, Mazgareanu S, Rother M. Preclinical characterisation of NSAIDs in ultradeformable carriers or conventional topical gels. Int $J$ Pharm. 2008;36:29-39.

15. Kang MJ, Eum JY, Park SH, et al. Pep-1 peptide-conjugated elastic liposomal formulation of taxifolin glycoside for the treatment of atopic dermatitis in NC/Nga mice. Int J Pharm. 2010;402:198-204.

16. Dragicevic-Curic N, Scheglmann D, Albrecht V, Fahr A. Development of different temoporfin-loaded invasomes - novel nanocarriers of temoporfin: characterization, stability and in vitro skin penetration studies. Colloid Surf B Biointerfaces. 2009;70:198-206.

17. Dragicevic-Curic N, Scheglmann D, Albrecht V, Fahr A. Temoporfinloaded invasomes: development, characterization and in vitro skin penetration studies. $J$ Control Release. 2008;127:59-69.

18. Subongkot T, Duangjit S, Rojanarata T, Opanasopit $P$, Ngawhirunpat T. Ultradeformable liposomes with terpenes for delivery of hydrophilic compound. J Liposome Res. 2012;22:254-262.

19. Subongkot T, Wonglertnirant N, Songprakhon P, Rojanarata T, Opanasopit P, Ngawhirunpat T. Visualization of ultradeformable liposomes penetration pathways and their skin interaction by confocal laser scanning microscopy. Int J Pharm. 2013;441:151-161.

20. Coderch L, Fonollosa J, Pera MD, Estelrich J, Maza ADL, Parra JL. Influence of cholesterol on liposome fluidity by EPR relationship with percutaneous absorption. $J$ Control Release. 2000;68:85-95.

21. Man D, Olchawa R, Kubica K. Membrane fluidity and the surface properties of the lipid bilayer: ESR experiment and computer simulation. J Liposome Res. 2010;20:211-218.

22. Anjos JLD, Alonso A. Terpenes increase the partitioning and molecular dynamics of an amphipathic spin label in stratum corneum membranes. Int J Pharm. 2008;350:103-112.

23. Haag SF, Fleige E, Chen M, et al. Skin penetration enhancement of coremultishell nanotransporters and invasomes measured by electron paramagnetic resonance spectroscopy. Int J Pharm. 2011;416:223-228. 
24. Lannone A, Rota C, Bergamini S, Tomasi A, Canfield LM. Antioxidant activity of carotenoids: an electron-spin resonance study on beta-carotene and lutein interaction with free radicals generated in a chemical system. J Biochem Mol Toxicol. 1998;12:299-304.

25. Dragicevic-Curic N, Friedrich M, Petersen S, et al. Assessment of fluidity of different invasomes by electron spin resonance and differential scanning calorimetry. Int J Pharm. 2011;412:85-94.

26. Yeom S, Shin BS, Han S. An electron spin resonance study of non-ionic surfactant vesicles (niosomes). Chem Phys Lipids. 2014; 181:83-89.

27. Keith A, Bulfield G, Snipes W. Spin-labeled neurospora mitochondria. Biophys J. 1970;10:618-629.
28. Tasi LM, Liu DZ, Chen WY. Microcalorimetric investigation of the interaction of polysorbate surfactants with unilamellar phosphatidylcholines liposomes. Colloids Surf A. 2003;213:7-14.

29. Chain E, Kemp I. The isoelectric points of lecithin and sphingomyelin. Biochem J. 1934;28:2052-2055.

30. Saesoo S, Sramala I, Soottitantawat A, Charinpanitkul T, Ruktanonchai UR. Enhanced stability and in vitro bioactivity of surfactant-loaded liposomes containing Asiatic Pennywort extract. J Microencapsul. 2010; 27:436-446.

31. Maghraby GME, Barry BW, Williams AC. Liposomes and skin: from drug delivery to model membranes. Eur J Pharm Sci. 2008;34: 203-222.

\section{Publish your work in this journal}

The International Journal of Nanomedicine is an international, peerreviewed journal focusing on the application of nanotechnology in diagnostics, therapeutics, and drug delivery systems throughout the biomedical field. This journal is indexed on PubMed Central, MedLine, CAS, SciSearch $\AA$, Current Contents ${ }^{\circledR} /$ Clinical Medicine,
Journal Citation Reports/Science Edition, EMBase, Scopus and the Elsevier Bibliographic databases. The manuscript management system is completely online and includes a very quick and fair peer-review system, which is all easy to use. Visit http://www.dovepress.com/ testimonials.php to read real quotes from published authors. 